\title{
Recommendations for centres of expertise in rare anaemias. The ENERCA White Book
}

\author{
Joan-Lluis Vives Corrons, ${ }^{1}$ María del Mar Mañú Pereira, ${ }^{1}$ Carlos Romeo-Casabona, ${ }^{2,3}$ \\ Pilar Nicolás, ${ }^{2,3}$ Béatrice Gulbis, ${ }^{4}$ Androulla Eleftheriou, ${ }^{5}$ Michael Angastiniotis, ${ }^{5}$ \\ Patricia Aguilar-Martínez, ${ }^{6}$ Paola Bianchi, ${ }^{7}$ Richard Van Wijk, ${ }^{8}$ Hermann Heimpel, ${ }^{9}$ \\ Barbara De la Salle, ${ }^{10}$ Andrea Mosca ${ }^{11}$
}

${ }^{1}$ Hospital Clinic, University of Barcelona, Spain; ${ }^{2}$ Interuniversity Chair in Law and the Human Genome, University of Deusto, Spain; ${ }^{3}$ University of the Basque Country, Provincial Government of Bizkay, Spain; ${ }^{4}$ Department of Clinical Biology \& Molecular Genetics; Clinical Chemistry Department, Hôpital Erasme; Université Libre de Bruxelles, Brussels, Belgium; ${ }^{5}$ Thalassaemia International Federation and Cyprus Thalassaemia Centre, Cyprus; ${ }^{6}$ Department of Haematology, Montpellier Hospital Center of Expertise on Rare Iron Disorders, Montpellier, France; ${ }^{7}$ Oncohaematology Unit, Physiopathology of Anemias Unit, Foundation IRCCS Ca' Granda Ospedale Maggiore Policlinico, Milan, Italy; ${ }^{8}$ Department of Clinical Chemistry and Haematology; University Medical Center Utrecht, The Netherlands; ${ }^{9}$ Department of Internal Medicine III and Centre of Rare Diseases, University of UIm, Germany; ${ }^{10}$ UK National External Quality Assessment Service, UK; ${ }^{11}$ Department of Physiopathology and Transplantation; University of Milan, Italy

\begin{abstract}
The Community added value of Centres of Expertise $(\mathrm{CoE})$ and European Reference Networks (ERN) is particularly high for rare diseases (RD) due to the rarity of these conditions, which implies both a small number of patients and scarcity of expertise within a single country. Gathering expertise at the European level is therefore, paramount in order to ensure equal access to accurate information, appropriate and timely diagnosis and high quality clinical care and follow up for patients with rare diseases. This applies particularly to rare anaemias due to the high number of different rare diseases that constitute this group. In this context, the European Network for Rare and Congenital Anaemias (ENERCA), co-financed by the European Commission, was created in 2002 with the aim of prevention and management of rare anaemias (RA) and the development and promotion of policies to improve the well-being of European Union citizens. The
\end{abstract}

Correspondence: Joan-Lluis Vives Corrons, Hospital Clinic, University of Barcelona, Spain

E-mail: jlvives@clinic.ub.es; enerca@enerca.org

Key words: rare anaemias; ENERCA; Europe; public health policies; haemoglobinopathies.

(C) Copyright J.-L. Vives Corrons et al., 2014

Licensee PAGEPress, Italy

Thalassemia Reports 2014; 4:4878

doi:10.4081/thal.2014.4878

This article is distributed under the terms of the Creative Commons Attribution Noncommercial License (by-nc 3.0) which permits any noncommercial use, distribution, and reproduction in any medium, provided the original author(s) and source are credited.
ENERCA White Book is a position paper, developed as a deliverable of the ENERCA (phase 3) project that intends to contribute to the creation of a ERN in RA (ERN-RA) by preparation of the recommendations and, in particular, the definition of the criteria that $\mathrm{CoE}$, local centres (LC) and their interrelations have to fulfil as healthcare providers. It has been nourished by all the activities that have been performed over the past ten years within the ENERCA framework. The White Book is addressed to authorities in charge of the identifying $\mathrm{CoE}$, as an essential requirement for the official recognition of the ERN, to European and national health authorities, Healthcare centres and health professionals, as well as to all other stakeholders interested in RA. It is also addressed to the patients, as a way to empower their community in this process. One particular characteristic of the White Book is the integration of the three main aspects of a CoE: a) ethical and legal frameworks to ensure the non-discrimination and non-stigmatisation of rare disease patients across Europe, within their sphere of competencies; b) clinical and laboratory frameworks for defining technical and quality criteria including scope, general and disease specific elements currently defined as technical and professional standards for the diagnosis, treatment and follow-up of patients with RA; and c) the expectations patients have of $\mathrm{CoE}$. Conceived as a working tool directed to a broad range of stakeholders, the White book has been designed and structured to be comprehensible even to non-technical and /or non-professional audiences. The reader will find an up-to-date description and epidemiological information on RA as well as the European Union background policies for defining CoE and ERN-RA. A working group was created with experts of different profiles, known as the European Working Group on Rare Anaemias (EGRA). In order to achieve its objectives, the methodology used by EGRA, was characterised by three main principles: Interdisciplinary, European coverage, and evidence-based principles. Work has been developed into four sequential steps: 1 . Analysis of the current situation of RA in Europe by healthcare professionals in order to identify the most relevant issues that have to be addressed by a centre in order for it to be recommended as CoE. 2. Preparation of questionnaires to perform sur- 
veys on how the relevant issues identified in step 1 can be translated into practical recommendations. 3. Analysis of the questionnaire results by face to face meetings, feedback and consensus evaluation, and 4. Preparation of a report on ENERCA policy recommendations for $\mathrm{CoE}$. This report is presented in a user-friendly format, easy to understand and available through the ENERCA website (www.enerca.org). Several important conclusions can be drawn from the ENERCA White Book, including the importance of laboratories involved in the diagnosis of RA, patient oriented and multidisciplinary care at the CoE, the need for coordination and cooperation within and outside the centre, the provision of information to patients and health professionals and the involvement of public authorities at the national and European levels. Official recognition of this structure and assurance of its long term sustainability will only be achieved if public authorities work hand by hand with both professionals experts in different disciplines and patients. Finally, the ENERCA White book aims to be a practical tool for health authorities of Member States (MS) that are preparing their national directory of formally designated CoE. For this, it is important that MS authorities recognise RA as an important health component to be included within the National Plans or Actions for Rare Diseases.

\section{Introduction}

Rare diseases (RDs) are probably the area in public health in which joint efforts among European Member States (MS) is most justified and crucial. This is because a common European approach would be more rational, efficient and effective than 27 individual National approaches. According to the European Commission (EC), a disease is considered "rare" in Europe when it affects less than 1 in 2000 individuals. The specificities of RDs, including a limited number of patients and the scarcity of knowledge and experience, certainly single them out as an extremely special area of very high European added-value. The fact that often no effective cures exist adds to the high level of pain and suffering endured by patients and their families. In addition, the lack of specific health policies for RDs and the scarce and scattered research performed in highly specialised laboratories and centres throughout the EU, makes it difficult of access clinical care and consequently leads to a delayed diagnosis. Therefore, a call for European and International cooperation, to ensure this knowledge is shared so that everyone can benefit from combined resources, has become an important public health challenge. The ongoing implementation of a better comprehensive approach to RDs has led to the development of appropriate public health policies, and important gains continue to be made with the increase of international cooperation and the development of new diagnostic and therapeutic procedures.

"Rare Anaemias" (RAs) constitute an important and relatively homogeneous group of RDs where "anaemia" is the first and most relevant clinical manifestation of the disease. This importance was recognised for first time by the EC in 2002 when it approved the co-financing of the DG-SANCO Project: "European Network for rare congenital diseases" (ENERCA). Interestingly, this project started shortly before the creation of the High Level Group (HLG) in 2004, which brought together experts in several areas of 20 expertise from all the MS. For RAs, this was a great advantage because it facilitated the gradual development of the ENERCA Project in parallel to the development of different HLG areas of action: a) patient safety and quality of care, b) health impact assessment and health systems, c)health technology assessment, d) European workforce for health professionals, e) European reference networks, f) information and e-health and, more recently, g) cross-border healthcare purchasing and provision.

Before ENERCA, RAs were almost unknown in Europe, including some health professionals, because in many cases, the cause of the anaemia was unknown and/or no treatment was available. Moreover, for many years anaemias, in general, have been underestimated by public health providers, due to their frequent misdiagnosis as iron deficiency anaemia, the most frequent cause of anaemia worldwide. ENERCA definitively changed this situation by the development of three consecutive Projects or Phases with a total duration of 10 years.

Accordingly, the creation of a ERN of CoE in RAs is a key strategy to improve the clinical management of these patients and to reduce health inequalities across the EU and tackling RDs through a Europewide network. This will accomplish one of the main objectives of the Commission's Work Plan for 2008, "putting citizens first", which includes improving patient safety and the quality of health services. For this, after 2012, the EC accepted co-financing a ENERCA extension (eENERCA) for the development of an e-health platform that will facilitate the diagnosis and clinical management of severe RAs through the use of new telediagnosis and telemedicine methodologies. As established by the Directive 2011/24/EU of 9 March 2011 on the application of patients' rights in cross-border healthcare, RD Networks are a very important tool that should be promoted within that general framework.

In this context, the ENERCA White Book is a policy document structured to be comprehensible beyond a technical and professional level. It has been prepared by a Working Group within ENERCA team (EGRA), and is addressed to a broad range of stakeholders in a friendly user format. The reader will find up-to-date information on RAs, including definitions, individual descriptions and epidemiological data, in addition to important information on the EU background policies for defining $\mathrm{CoE}$ and ERN. An important objective has been to provide a practical document containing specific material and methodology that can be used to establish a consensus on what criteria should be considered in the process of identification and designation of $\mathrm{CoE}$ by national health authorities. The methodology used is presented in detail in a separate chapter and explained by integrating three interdisciplinary approaches: a) a legal and ethical perspective; b) a clinical and laboratory approach concerning specific methodology and quality criteria for diagnosis, treatment and clinical follow up of each rare anaemia; and c) patient expectations. In fact, the document is expected to be a dynamic working tool that can be modified over time, if necessary.

\section{Materials and Methods}

To prepare the CoE recommendations for RAs a Working Group was created within ENERCA (EGRA), integrating the following profiles: a) physicians and health care professionals, b) molecular biologists and basic scientists, c) experts in legal and ethical matters d) patient associations. For achieving the final objective the following principles were followed:

- Interdisciplinary, integrating persons from different sources of knowledge: clinical and laboratory professionals, experts in ethical and legal issues and patients.

- European coverage including experts from eight different European countries: Belgium, Cyprus, France, Germany, Italy, Spain, The Netherlands and United Kingdom. Involvement of other MS was assured by broadening the different surveys performed, seeking experts from different countries and the involvement of TIF, the international umbrella for patient associations.

- Evidence based, ensuring, as much as possible, a realistic approach for achieving the final results by using the current situation of real practice as starting point.

Using these principles, the following consecutive steps were covered:

- Analysis of current situation in Europe, in order to identify and select the "hot spot" topics (most relevant issues) to be addressed into the recommendations for $\mathrm{CoE}$ recognition.

- Using the results of the this analysis serveral surveys were undertak- 
en in order to check how the "hot spots" can be translated into practical recommendations.

- Evaluation of the responses to the survey's questionnaires to achieve a final consensus.

- Preparation of final recommendations on criteria to recognise CoE.

\section{Results}

The consensus recommendations are the outcome of a proposal of general agreement between the different ENERCA partners involved in different process of labelling or recognition as centres of expertise for rare and very rare anaemias in their respective countries; ditto for the local centres.

For countries where no such process is running, this consensus could be an example, for countries where this process is running, as this consensus offers an ideal level of services to be provided, it may serve to raise the current level.

Based on the three reports resulting from the surveys analysis, proposals of recommendations were elaborated and circulated in order to be discussed in a multidisciplinary way. The interactions between the different proposals were a key point to be analyzed. In addition, two external advisers were invited to review the draft.

Finally a set of recommendations were proposed as the minimal required criteria for the recognition of a healthcare provider as a Centre of Expertise (CEs) in one of the following categories:

1st. "Centre of expertise" is a centre with an appropriate capacity to address the complex and diverse conditions of RAs within a multidisciplinary approach. A centre of expertise provides expert advice, produces guidelines and has links with other centres of expertise building a European Reference Network.

2nd. "Local centre" is a centre that offers health care in a defined catchment area. Local centres are bounded to a centre of expertise

\section{Legal and ethical recommendations}

1. General: security measures for data files, electronic clinical records, ethical and legal training for professionals and quality requirements of centres in order to transfer data and samples. 2. Diagnosis and treatment: period for the storage of "inactive" patient data, transfer of clinical data and samples, information and consent for the transfer of identified data or samples, information about health care in other centres, procedure to transfer of data /samples / patients, requirement for professional proof or evidence of expertise and Quality standards regulations 3. Research: patient consent for the use of samples or data for research purposes

\section{Laboratory practice recommendations}

1. Laboratory accreditation and necessary resources, 2 . Reference laboratories identification and participation in External Quality Assessment Schemes (EQAS), 3. Professional expertise and experience of the laboratory practice, 4 . Strong contribution to research, 5 . Close collaborative links for epidemiological surveillance, algorithm testing, standard operating procedure (SOP) preparation and use of guidelines for the standardised reporting of antenatal screening results and documented risk management policy

\section{Clinical practice and patient's follow-up recommendations}

1. Capacity for detecting and treating severe disease complications, 2. Capacity to provide expert case management in chronic blood disorders including:

- Availability of a blood bank

- Availability of orphan drugs
- Availability of a coordinated multi-disciplinary team of specialists

- Free treatment

- Holistic care should be provided

- Maintaining a dedicated clinical record

- Specialised staff to follow

- Specialised facilities for patient care and drug administration

3. Provide expert advice on genetic counselling, appropriate staff to patient ratio, evidence, proof of expertise, implementation of outcome measures and quality control 4. Contribute to generate an agreed registry between $\mathrm{CoE}$ and local centres, 5 . Monitoring and evaluating indicators of outcomes, 6. Provide relevant services with efficient activity and high level expertise and experience at a sustained level of quality.7. Capacity of establish close links and collaboration with other expert national and international centres and 8 . capacity to network and special services for patients

\section{Patient expectations recommendations}

1. Early diagnosis and management of complications. This is a lifesaving requirement that any centre of expertise must be able to provide and also use to support local or secondary centres. This important requirement implies both clinical experiences, following management guidelines but also having the technical means for specialised tests. 2 . Availability of specialists in the expert centre that has networking arrangements with local centres. 3. Expertise for providing information and genetic counselling based on the interpretation and understanding of the laboratory diagnosis, intimate knowledge of the disease consequences, available treatments as well as the choices offered to at-risk couples, following accepted principles of counselling. Accordingly this service has to be provided by experts and not by general staff who may easily mislead affected individuals or couples. For this a proof of expertise is important and indicators, such as outcome measures, are recommended by EUCERD. In some countries such as UK, there is an accreditation system and a team of experts, including expert patients assessing advices in the treatment centres.

\section{Discussion}

Cooperation and knowledge sharing between expert centres has proven to be a very efficient approach to dealing with rare diseases in Europe. Accordingly, cost-effective programs for RA will be only possible if a tight cooperation exists between European expert centres. These programs will be developed on the basis of optimization of the resources currently existing in each member state participating in ENERCA Project through their correspondent beneficiary. One of Project's aims is to ensure that RAs are adequately coded and traceable in all healthy information systems in order to allow the recognition of each disease in the national healthcare and reimbursement systems based on the International Classification of Diseases (ICD). Gathering expertise at European level is therefore paramount to ensure equal access to accurate information, appropriate and timely diagnosis and high quality care for rare anaemias patients. Moreover, increasing Healthy Life Years by preventing disease and promoting policies that lead to a healthier way of life is important for the well-being of EU citizens and helps to meet the challenges of the Lisbon decision as regards the knowledge society and the sustainability of public finances, which are under pressure from rising health care and social security costsCooperation and knowledge sharing between $\mathrm{CoE}$ has proven to be a very efficient approach to deal with rare diseases in Europe. The EC could follow a multidisciplinary approach to care in order to address the complex and diverse conditions implied in this group of rare diseases called rare anaemias.

The existence of ERNs in RDs is a particular Community added value 
ERNs due to the scarce number of patients and expertise within a single country. The ERN will identify needs and priorities for basic, clinical translational and social research in the fields of the different rare anaemias categories: a) Sickle cell disease (SCD), b) Thalassaemia syndromes and d) Very Rare Anaemias (e.g. hereditary or acquired erythropoietic failure, and hereditary or acquired RBC defects). This will facilitate ENERCA fostering all these activities and promote interdisciplinary cooperative approaches to the complementarily addressed through national and Community programmes. Dissemination and sustainability of the ENERCA web as well as its activities and services, will be assured by the promotion of links with other associations and stakeholders (public institutions, public health authorities, pharmaceutical companies.

The Community added value of ERNs is particularly high for rare diseases due to the rarity of these conditions, which implies both a limited number of patients and a scarce of expertise within a single country. Accordingly, the consolidation of the ERN for RAs will allow the health professionals to share the best practices on diagnostic tools and medical care as well as education and social care regardless of their country of origin, an easy access to recognized $\mathrm{CoE}$ in each RA category. Moreover, the establishment of a European registry of RAs will facilitate the epidemiological surveillance of these diseases across the EU, very important in EU countries with high immigration input and where the former "very rare anaemias" have become familiar (Sickle-cell anaemia is an example). Comparable epidemiological data at EU level will promote the establishment of specific actions for primary prevention of prevalence increase of imported RAs in such European countries where it is higher than expected due to immigration impact. Accordingly, ENERCA will promote the establishment of prevention programs for haemoglobinopathies that will be of great help for the establishment of an early diagnosis of the diseases and its primary prevention. In addition, ENERCA will identify ongoing research in $R A s$ and research resources in the national and Community frameworks in order to establish the state of the art, to assess the research landscape and improve the coordination of Community, national and regional programmes for RAs research. One of Project's priorities in this field will be foster the participation of experts and national researchers in the identification of needs and priorities for basic, clinical transnational and social research in rare anaemias; trans-national research on pathophysiology of RA, including molecular genetics diversity and optimal management for a timely diagnosis. Communication between all implicated experts will facilitate the compilation of data from patients coming from a large number of countries and this will be very useful for research because the data on RA that can be obtained in each individual country are not high enough for obtaining meaningful conclusions.

Finally, ENERCA will contribute to the harmonization of the haematology and paediatric (haematology) specialities curricula throughout the EU (re: "European haematology curriculum/passport" accessible on the European Haematology Association website). This will be achieved by fostering the active participation of expert centres in the organization of training courses on biological and clinical aspects of these medical specialties. The permanent contact with the European School of Haematology (ESH), one of the ENERCA 3 associated partners, will be a guarantee of success. Preparation of educational material will allow patients' access to specific information on RA in their own language. Consult patients and patients' representatives on the policies in the field of rare anaemias will facilitate patients' access to updated information on rare anaemias. This will increase the awareness and knowledge of patient's and their relatives about RA. Moreover, the promotion of activities performed by patient organizations and/or associations will increase their empowerment in the elaboration of policies and in the decision making processes.

\section{Conclusions}

Gathering expertise at the European level is paramount to ensure equal access to healthcare services (accurate information, appropriate and timely diagnosis and high quality care) for patients with rare diseases. This is especially important for RAs, due to the high number of RDs included under the name of "Very Rare Anaemias" or "undiagnosed rare anaemias". ENERCA, as a pilot network funded by the European Commission in 2002 , has contributed, during more than 10 years, to the establishment of a solid framework providing experience and advice in RAs. Moreover, ENERCA White Book is the first multi-disciplinary approach to combine clinical, technical , legal and patient's expectations recommendations that the $\mathrm{CoE}$ in RAs have to fulfil in to become nodes of the future ERN-RA. The involvement of national authorities is essential for the development of networks in two main areas: the recognition of $\mathrm{CoE}$ and the ERN sustainability. The strategy should involve both aspects, as stated in the Council Recommendation of 2009.The work done regarding recommendations included in this White Book is a practical tool that MS can use for the recognition of $\mathrm{CoE}$ in their national plans. The methodology used could be transposed to other rare diseases and MS should take into account the efforts already made by ENERCA and ENERCA is committed to present the White Book to National Authorities responsible of National Plans for Rare Diseases.

This pioneer activity of ENERCA Project is an added value that may be useful for any other network on rare chronic diseases. Its efficiency as a pilot network has been demonstrated in relation to all its objectives one of these is the final characterization on the more than $20 \%$ of RAS that still remain undiagnosed. Successful achievements of ENERCA's 10 years of life are the following.

\section{Best practices guidelines platform}

ENERCA experts are deeply committed to the preparation of clinical and laboratory recommendations including all aspects of clinical practice. The cumulative knowledge and experience of these experts represents a resource that must be upgraded to the status of guidelines.

\section{External Quality Assessment Schemes (EQAS) for RAs diagnostic tests}

ENERCA has contributed to auditing laboratory practices by promoting the use of highly specialized tests necessary for the diagnosis of RAs . For the very rare diseases diagnostic tests, a European approach is required to reach a minimal critical mass of laboratories performing a particular EQAS. ENERCA has identified some gaps in this context and has promoted the creation of new EQAS to assure high quality diagnosis for patients with very rare anaemias such as enzymopathies and red blood cell membrane defects. Networking has allowed an easier identification of laboratories working on the same diseases, facilitated audit and sharing of best practice and information on EQA across national boundaries.

\section{Registries of patients}

ENERCA has a long time experience in the creation of registries for RAs at both national and international levels, and has participated in several EC policies in this direction. This is essential to achieve the objectives of a ERN in RAs, in both clinical practice and research and there have been taken into account all of the parameters involved, including patient rights, standardisation of procedures (including diseases codification), professional involvement and technical support.

\section{Educational activities}

ENERCA has undertaken a strong activity in tis field by using networking has a huge potential tool for teaching and training. Accordingly, successful meetings (workshops and Symposia) have been 
organised at national and international levels focused on specific aspects of RAs. Networks are also a useful tool to assure the inclusion of RDs in continuing medical education programmes.

\section{Telemedicine and telexpertise}

ENERCA is currently developing three new platforms based on the information and communication technologies (ICT) that are: eRegistry, e-Learning and e-Health . The later will serve the needs of the multi-disciplinary teams that are necessary for the diagnosis and clinical care support for RAs, especialy when severe complications affect vital organs. Networks shall be supported through information systems with interoperability including electronic health records (EHR), ehealth systems and the sharing of data. Electronic networking is the most cost-effective method of collaborative exchange; however, the need for patient travel and for local assessment by an expert should not be completely eliminated in order to achieve the best level of health care provision. Cross border health, as a recognised right of European citizens, must be respected and supported.

\section{Research in multi-centre settings}

ENERCA is promoting research through the involvement of partners in research projects from FP7 and Horizon 2020 calls. Joint research activities are the only way to discover new causes leading to RA in the substantial group of patients that remain undiagnosed. Moreover research activity is also part of $\mathrm{CoE}$ and $\mathrm{EC}$ networking by participating in clinical trials on in protocols for new drugs investigation.

\section{Networking of $\mathrm{CoE}$}

This is a pivotal activity of ENERCA undertaken by the EGRA in the preparation of ENERCA White Book. It is well known that differences exist between national regulations and, in the same way, differences can be also found regarding sample management and biobanking. These differences may become, in some cases, a barrier to networking. Accordingly, networking is imperative for the exchange of knowledge, patient's samples and personal data transfer in both clinical management and research, as well as in legal and ethical issues. Providing patients with adequate information before they give their consent to the clinical procedure or involvement in research seems to be a key way to overcome this problem. This information should include the possibility of integrating clinical data in a network and its consequences, as well as the description of the future research foreseen. The importance of this harmonisation will rise, taking into account the coming transposition of Directive 2011/24/EU of the European Parliament and of the Council of 9 March 2011 regarding the application of patients' rights in cross border healthcare. Professional oversight and coordination of the network is required to ensure the maintenance of agreed standards and the dissemination of information to all European MS

\section{Patient's empowerment}

This is also an important objective of ENERCA, and it has resulted in several patient-based recommendations for the recognition of $\mathrm{CoE}$. Involving the patient community is essential in the context of RD since patient's contribution leads to a more complete and realistic approach of the disease and assures that the quality of care matches patient expectations.

\section{Final remarks}

In order to assure long term sustainability of the network, there is a strong need for official recognition of this structure. The major prerequisite for the development of national plan/strategies on RDs, including CEs and ERNs, is financial support for their sustainability.
The existing heterogeneity between MS in health infrastructures and technical capacities of NHS systems to support such programmes, and the resources available at MS level to allocate for them, has to be considered because networks represent, not only, an academic exercise for the research community, but rather to completely integrate them within a public health strategy. This is why the EC also plays an important role for the international dimension of these strategies. In this direction, the EUCERD Join Action is developing its activity in two main directions: a) the establishment of a common framework for the creation of ERNs for RD and b) the implementation of mechanisms to assure their sustainability.

\section{References}

Commission Communication, Rare Diseases Europe's Challenge. Available from: http://ec.europa.eu/health/ph_threats/non_com/ docs/rare_com_en.pdf

Council Recommendation (2009/C 151/02) of 8 June on an action in the field of rare diseases. Available from: http//eur-lex.europa.eu/Lex UriServ/LexUriServ.do?uri=0J:C:2009:151:0007:0010:EN:pdf

Directive (EC 2011/24/EU) of the European Parliament and of the Council on the application of patients' rights in cross-border health care. Available from: http://eur-ex.europa.eu/LexUriServ/LexUri Serv.do?uri=0J:L:2011:088:0045:0065:EN:pdf

ENERCA Recommendations for Centres of Expertise in Rare Anaemias: A White Book. Ed. Drug Pharma 2014. Available from: http://www. enerca.org

EUCERD Report: Preliminary analysis of the experiences and outcomes of ERNs for rare diseases (May 2011). Available from: http://www. eucerd.eu/upload/file/Reports/ERN2011Analysis.pdf

EUCERD Workshop Report: Centres of expertise and European Reference Networks for Rare Diseases (8-9/12/2010). Available from: http://www.eucerd.eu/upload/file/WorkshopReport/EUCERD WorkshopReportCECERN.pdf

EUCERD Workshop Report: National centres of expertise for rare diseases and networking between centres of expertise for rare diseases (21-22/03/2011). Available from: http://www.eucerd.eu/upload /file/EUCERDReport220311.pdf

EUROPEAN COMISSION: Results of Public Consultation on the implementation of European Reference Networks (Directive 2011/24/EU of the European Parliament and of the Council of 9 March 2011 on the application of patients' rights in cross-border healthcare) Brussels, June 2013. Available from: http://ec.europa.eu/health/ cross_border_care/consultations/cons_implementation_ern_en.htm

EUROPLAN: Recommendations for the Development of National Plans and Strategies for Rare Diseases. Available from: http://www.europlanproject.eu/public/contenuti/files/Guidance_Doc_EUROPLAN_20100601_final.pdf

RDTF Report: Centres of Reference for Rare Diseases in Europe State-of-the-art in 2006 and Recommendations of the Rare Diseases Task Force (September 2006). Available from: http://www. eucerd.eu/upload/file/Publication/RDTFECR2006.pdf

RDTF Report: European Reference Networks in the field of Rare Diseases: State of the art and Future Directions (July 2008). Available from: http://www.eucerd.eu/upload/file/Publication/RDTFERN2008.pdf

RDTF Report: Overview of Current Centres of Reference on rare diseases in the EU (September 2005). Available from: http://www. eucerd.eu/upload/file/Publication/RDTFECR2005.pdf

Recommendations on Quality Criteria for Centres of Expertise for Rare Diseases in Member States. Available from: http://www.eucerd.eu/? post_type $=$ document\&p $=1224$

Work of the High Level Group on Health Services and Medical Care during 2005. Available from: http://ec.europa.eu/health/archive/ph_ overview/co_operation/mobility/docs/highlevel_2005_013_en.pdf 